\title{
ON THE ASYMPTOTIC FORMULA IN WARING'S PROBLEM: ONE SQUARE AND THREE FIFTH POWERS
}

\author{
JÖRG BRÜDERN \\ Mathematisches Institut, Bunsenstrasse 3-5, \\ D-37073 Göttingen, Germany \\ e-mail:bruedern@uni-math.gwdg.de
}

(Received 27 January 2014; accepted 12 May 2014; first published online 18 December 2014)

2010 Mathematics Subject Classification. 11P55.

1. Let $r(n)$ denote the number of representations of the natural number $n$ as the sum of one square and three fifth powers of positive integers. A formal use of the circle method predicts the asymptotic relation

$$
r(n)=\frac{\Gamma\left(\frac{3}{2}\right) \Gamma\left(\frac{6}{5}\right)^{3}}{\Gamma\left(\frac{11}{10}\right)} \mathfrak{s}(n) n^{\frac{1}{10}}(1+o(1)) \quad(n \rightarrow \infty) .
$$

Here $\mathfrak{s}(n)$ is the singular series associated with sums of a square and three fifth powers, see (13) below for a precise definition. The main purpose of this note is to confirm (1) in mean square.

THEOREM 1. One has ${ }^{1}$

$$
\sum_{m \leq n}\left(r(m)-\frac{\Gamma\left(\frac{3}{2}\right) \Gamma\left(\frac{6}{5}\right)^{3}}{\Gamma\left(\frac{11}{10}\right)} \mathfrak{s}(m) m^{\frac{1}{10}}\right)^{2} \ll n^{\frac{6}{5}}(\log n)^{\varepsilon-1} .
$$

Further, the singular series $\mathfrak{\mathfrak { s }}(m)$ is real and satisfies the inequalities

$$
1 \ll \mathfrak{s}(m) \ll(\log \log m)^{3} .
$$

Let $\delta>0$. It follows from (2) that

$$
\left|r(m)-\frac{\Gamma\left(\frac{3}{2}\right) \Gamma\left(\frac{6}{5}\right)^{3}}{\Gamma\left(\frac{11}{10}\right)} \mathfrak{s}(m) m^{\frac{1}{10}}\right| \leq m^{\frac{1}{10}}(\log 2 m)^{-\delta}
$$

holds for all but $O\left(n(\log n)^{2 \delta+\varepsilon-1}\right)$ of the natural numbers $m$ not exceeding $n$. In particular, for all but $O\left(n(\log n)^{\varepsilon-1}\right)$ such $m$ one has $r(m) \gg m^{1 / 10}$.

For natural numbers $k$, s with $k \geq 3$, let $r_{k, s}(n)$ denote the number of representations of $n$ as the sum of a square and $s$ positive $k$-th powers. The problem of providing an asymptotic formula for $r_{k, s}(n)$ has a long history, apparently beginning with the work of Stanley [11]. Once again through a formal use of the circle method, one is led to predict such an asymptotic formula with leading term of size about $n^{\frac{s}{k}-\frac{1}{2}}$

\footnotetext{
${ }^{1}$ Statements involving the letter $\varepsilon$ are valid for any positive value of $\varepsilon$, numbers implicit in Vinogradov or Landau symbols may depend on $\varepsilon$. This convention is applied throughout this article.
} 
whenever $2 s>k$. We are far from a proof, it seems, and at best one may attempt a demonstration for almost all $n$. An analogue of our Theorem 1 for $r_{3,2}(n)$ is implicit in the work of Davenport and Heilbronn [4], and an average treatment of $r_{4,3}(n)$ is also possible by an extension of Exercise 2.8.5 of Vaughan [16]. Theorem 1 adds the case $k=5$ to the stock of examples where the smallest $s$ with $2 s>k$ has been successfully treated in mean square. We mention in passing that rather more $k$-th powers are required if one insists that the proposed asymptotic formula for $r_{k, s}(n)$ be valid for all $n$. Such is only known for $k=3, s \geq 6$ (Sinnadurai [10], Hooley [8]), $k=4, s \geq 10$ and $k=5, s \geq 17$ (Brüdern and Kawada [3]).

We now turn to problems involving two squares. Let $R(n)$ denote the number of representations of $n$ as the sum of two squares and six fifth powers of natural numbers, and let $\mathfrak{S}(n)$ be the singular series associated with such representations (see (24) for a definition).

THEOREM 2. One has

$$
R(n)=\frac{5}{24} \pi \Gamma\left(\frac{6}{5}\right)^{5} \mathfrak{S}(n) n^{\frac{6}{5}}+O\left(n^{\frac{6}{5}}(\log n)^{\varepsilon-1}\right) .
$$

Further, there are positive numbers $c, c^{\prime}$ with $c<\mathfrak{S}(n)<c^{\prime}$ for all natural numbers $n$.

This shows that all large numbers are the sum of two squares and six fifth powers, a conclusion that the author had obtained earlier [1, Theorem 4].

It may be of interest to compare Theorem 2 with other estimates for the number $R_{k, s}(n)$ of representations of $n$ as the sum of two squares and $s$ positive $k$-th powers. Our focus is on results valid for all $n$. There is a familiar square root cancellation barrier that places the limit $s>k$ on current technology for an asymptotic formula. For $R_{3,4}(n)$ and $R_{4,5}(n)$ such formulae can be found in work of Hooley [8] and Vaughan ([12] and [16], Exercise 2.8.5). Our Theorem 2 adds the case $k=5, s=6$ to this short list of examples where the barrier has been reached. Of course, one expects an asymptotic formula for $R_{k, s}(n)$ for all $s \geq 1$. In this direction, Hooley's spectacular demonstration of the anticipated formula for $R_{3,3}(n)$ stands out as a landmark on the square root cancellation barrier [9]. Continuing the line of thought in the discussion preceding Theorem 2, one might ask whether the asymptotic formula for $R_{k, 1}(n)$ is valid in mean square. However, this is yet another story to which we intend to return shortly.

For a thorougher account of Waring's problem with unlike powers the interested reader may consult the work of Hooley [8], chapter 8 of Vaughan [16] and the extensive references therein, and the recent papers of Wooley [18] and Friedlander and Wooley [5].

2. The proofs of both theorems are largely routine applications of the circle method, save for the minor arc treatment that features elements more typical for major arcs. The method rests on pruning devices that originate with work of Vaughan $[13,14]$ and the author [2]. We formulate a lemma that encapsulates all difficulties and then proceed to demonstrate it with two pruning steps.

In the interest of brevity, we write

$$
\delta=10^{-5}, \quad \eta=\delta^{2}, \quad L=\log n .
$$

For $0 \leq a \leq q,(a ; q)=1$ and $1 \leq q \leq n^{\delta}$, let

$$
\mathfrak{M}(q, a)=\left\{\alpha \in[0,1]:\left|\alpha-\frac{a}{q}\right| \leq n^{\delta-1}\right\},
$$


and let $\mathfrak{M}$ denote the union of these intervals. Finally, let $\mathfrak{m}=[0,1] \backslash \mathfrak{M}$. With $e(\alpha)$ as a shorthand for $\exp (2 \pi i \alpha)$, we introduce the Weyl sum

$$
f_{l}(\alpha)=\sum_{x \leq n^{1 / l}} e\left(\alpha x^{l}\right)
$$

LEMMA 1. One has

$$
\int_{\mathfrak{m}}\left|f_{2}(\alpha) f_{5}(\alpha)^{3}\right|^{2} \mathrm{~d} \alpha \ll n^{\frac{6}{5}} L^{\varepsilon-1}
$$

We begin the proof with recalling Dirichlet's theorem, asserting that any $\alpha \in[0,1]$ is contained in at least one of the intervals

$$
I_{1}(q, a)=\left\{\alpha \in[0,1]:|q \alpha-a| \leq n^{-\frac{1}{2}}\right\}
$$

with $0 \leq a \leq q,(a ; q)=1$ and $1 \leq q \leq n^{\frac{1}{2}}$. Meanwhile, the sets

$$
I_{2}(q, a)=\left\{\alpha \in[0,1]:|q \alpha-a| \leq \frac{1}{2} n^{-\frac{1}{2}}\right\}
$$

with $a, q$ as before are pairwise disjoint ([16], Exercise 1.5.3). It follows that there exist intervals $I(q, a)$ with $I_{2}(q, a) \subset I(q, a) \subset I_{1}(q, a)$ such that $[0,1]$ is the disjoint union of the $I(q, a)$, with $a, q$ still as before. Now define the function $\Upsilon:[0,1] \rightarrow[0,1]$ by

$$
\Upsilon(\alpha)=(q+n|q \alpha-a|)^{-1} \text { for } \alpha \in I(q, a) .
$$

Theorem 4 of Vaughan [17] shows that

$$
f_{2}(\alpha)^{2} \ll n \Upsilon(\alpha)+n^{\frac{1}{2}} \ll n \Upsilon(\alpha)
$$

holds uniformly for $\alpha \in[0,1]$, whence

$$
\int_{\mathfrak{m}}\left|f_{2}(\alpha) f_{5}(\alpha)^{3}\right|^{2} \mathrm{~d} \alpha \ll n \int_{\mathfrak{m}} \Upsilon(\alpha)\left|f_{5}(\alpha)\right|^{6} \mathrm{~d} \alpha .
$$

We estimate the contributions from two distinct parts of $\mathfrak{m}$ by different methods. Let

$$
\mathfrak{N}(q, a)=\left\{\alpha \in[0,1]:|q \alpha-a| \leq n^{\eta-\frac{3}{5}}\right\}
$$

and let $\mathfrak{N}$ denote the union of these intervals with $0 \leq a \leq q,(a ; q)=1$ and $1 \leq q \leq$ $n^{\frac{2}{5}+\eta}$. Put $\mathfrak{n}=[0,1] \backslash \mathfrak{N}$ and note that $\mathfrak{m}$ is the union of $\mathfrak{n}$ and $\mathfrak{N} \cap \mathfrak{m}$. The main difficulty is with the set $\mathfrak{n}$ where savings are only on the logarithmic scale. In preparation for the analysis of this set, we observe that for $0 \leq a \leq q,(a ; q)=1$ and $1 \leq q \leq n^{\frac{2}{5}+\eta}$, one has $\mathfrak{N}(q, a) \subset I_{2}(q, a)$. Hence, whenever $I(q, a)$ is defined, we write $I^{\prime}(q, a)=I_{1}(q, a)$ if $n^{\frac{2}{5}+\eta}<q \leq n^{\frac{1}{2}}$, and $I^{\prime}(q, a)=I_{1}(q, a) \backslash \mathfrak{N}(q, a)$ if $q \leq n^{\frac{2}{5}+\eta}$. Then $\mathfrak{n}$ is contained in the 
union of the $I^{\prime}(q, a)$. It follows that

$$
\begin{aligned}
\int_{\mathfrak{n}} \Upsilon(\alpha)^{2}\left|f_{5}(\alpha)\right|^{4} \mathrm{~d} \alpha & \leq \sum_{\substack{1 \\
q \leq n^{\frac{1}{2}}}} \sum_{\substack{a=1 \\
(a ; q)=1}}^{q} \int_{I^{\prime}(q, a)} \Upsilon(\alpha)^{2}\left|f_{5}(\alpha)\right|^{4} \mathrm{~d} \alpha \\
& \leq \sum_{q \leq n^{\frac{1}{2}}} q^{-2} \int_{\mathfrak{P}(q)}(1+n|\beta|)^{-2} \sum_{\substack{a=1 \\
(a ; q)=1}}^{q}\left|f_{5}\left(\frac{a}{q}+\beta\right)\right|^{4} \mathrm{~d} \beta
\end{aligned}
$$

where $\mathfrak{P}(q)=[-1,1]$ for $n^{\frac{2}{5}+\eta}<q \leq n^{\frac{1}{2}}$, and $\mathfrak{P}(q)=\left\{\beta: q^{-1} n^{\eta-\frac{3}{5}} \leq|\beta| \leq 1\right\}$ for $q \leq$ $n^{\frac{2}{5}+\eta}$. At this point, we made crucial use of the fact that the sets $I^{\prime}(q, a)-\frac{a}{q}$ are independent of $a$.

Let $\psi(h)$ denote the number of solutions of $x_{1}^{5}+x_{2}^{5}-x_{3}^{5}-x_{4}^{5}=h$ in natural numbers $x_{j}$ with $x_{j} \leq n^{\frac{1}{5}}$. Then $\psi(h)=0$ whenever $|h|>2 n$, and one finds that

$$
\left|f_{5}(\alpha)\right|^{4}=\sum_{|h| \leq 2 n} \psi(h) e(\alpha h) .
$$

This allows us to write

$$
\sum_{\substack{a=1 \\(a ; q)=1}}^{q}\left|f_{5}\left(\frac{a}{q}+\beta\right)\right|^{4}=\sum_{|h| \leq 2 n} \psi(h) e(\beta h) \sum_{\substack{a=1 \\(a ; q)=1}}^{q} e\left(\frac{a h}{q}\right) .
$$

The inner sum here is known as Ramanujan's sum, and one has [6, Theorem 272]

$$
\left|\sum_{\substack{a=1 \\(a ; q)=1}}^{q} e\left(\frac{a h}{q}\right)\right| \leq(q ; h) .
$$

Consequently, we now infer that

$$
\sum_{\substack{a=1 \\(a ; q)=1}}^{q}\left|f_{5}\left(\frac{a}{q}+\beta\right)\right|^{4} \leq \sum_{|h| \leq 2 n} \psi(h)(q ; h)
$$

and further

$$
\int_{\mathfrak{n}} \Upsilon(\alpha)^{2}\left|f_{5}(\alpha)\right|^{4} \mathrm{~d} \alpha \leq \sum_{|h| \leq 2 n} \psi(h) \sum_{q \leq n^{\frac{1}{2}}} \frac{(q ; h)}{q^{2}} \int_{\mathfrak{P}(q)}(1+n|\beta|)^{-2} \mathrm{~d} \beta .
$$

By Theorem 1 of Hooley [7], one finds that $\psi(0) \ll n^{\frac{2}{5}}$. Hence, the contribution of $h=0$ to the sum in (6) does not exceed

$$
\ll n^{\frac{2}{5}} \sum_{q \leq n^{\frac{1}{2}}} q^{-1} \int_{-1}^{1}(1+n|\beta|)^{-2} \mathrm{~d} \beta \ll n^{-\frac{3}{5}} L .
$$

For terms with $h \neq 0$ we consider separately the cases where $n^{\frac{2}{5}+\eta}<q \leq n^{\frac{1}{2}}$, and where $q \leq n^{\frac{2}{5}+\eta}$. In the first case, the integral on the right hand side of (6) is $O\left(n^{-1}\right)$ 
while in the second case we have the superior bound $O\left(q n^{-\eta-\frac{7}{5}}\right)$. Therefore, the part of (6) where $h \neq 0$ and $n^{\frac{2}{5}+\eta}<q \leq n^{\frac{1}{2}}$ contributes

$$
\ll n^{-1} \sum_{1 \leq|h| \leq 2 n} \psi(h) \sum_{q>n^{\frac{2}{5}+\eta}} \frac{(q ; h)}{q^{2}} \ll n^{-\frac{7}{5}-\eta} \sum_{1 \leq|h| \leq 2 n} \psi(h) \mathrm{d}(h) ;
$$

here $\mathrm{d}(h)$ is the number of divisors of $h$. Since $\mathrm{d}(h) \ll n^{\eta}$ holds for all relevant $h$, one infers from (4) with $\alpha=0$ that the above does not exceed

$$
\ll n^{-\frac{7}{5}} \sum_{h \in \mathbb{Z}} \psi(h) \ll n^{-\frac{3}{5}} .
$$

Similarly, the part of (6) where $h \neq 0$ and $q \leq n^{\frac{2}{5}+\eta}$ contributes

$$
\ll n^{-\frac{7}{5}-\eta} \sum_{1 \leq|h| \leq 2 n} \psi(h) \sum_{q<n} \frac{(q ; h)}{q} \ll n^{-\frac{3}{5}} .
$$

On collecting together, it follows from (6) that

$$
\int_{\mathfrak{n}} \Upsilon(\alpha)^{2}\left|f_{5}(\alpha)\right|^{4} \mathrm{~d} \alpha \ll n^{-\frac{3}{5}} L
$$

As a special case of Theorem B of Vaughan [15], one has

$$
\int_{0}^{1}\left|f_{5}(\alpha)\right|^{8} \mathrm{~d} \alpha \ll n L^{\varepsilon-3}
$$

On combining the last two estimates via Schwarz's inequality, we deduce that

$$
\int_{\mathfrak{n}} \Upsilon(\alpha)\left|f_{5}(\alpha)\right|^{6} \mathrm{~d} \alpha \ll n^{\frac{1}{5}} L^{\varepsilon-1}
$$

as desired.

It remains to examine the set $\mathfrak{N} \cap \mathfrak{m}$. This is rather simpler than the argument above. In fact, a straightforward adaptation of the method leading to (7) readily delivers the bound

$$
\int_{\mathfrak{N}} \Upsilon(\alpha)\left|f_{5}(\alpha)\right|^{4} \mathrm{~d} \alpha \ll n^{-\frac{1}{5}+\eta+\varepsilon} .
$$

Alternatively, this estimate also follows from Lemma 2 of Brüdern [2]. Weyl's inequality ([16, Lemma 2.4]) yields $f_{5}(\alpha) \ll n^{\frac{1}{5}-\frac{\delta}{16}+\varepsilon}$, so that we now have

$$
\int_{\mathfrak{N} \cap \mathfrak{m}} \Upsilon(\alpha)\left|f_{5}(\alpha)\right|^{6} \mathrm{~d} \alpha \ll n^{\frac{1}{5}-\eta} .
$$

The lemma follows from this, (8) and (3).

We do not claim a great degree of novelty for the method used here. In particular, the deduction of the critical estimate (7) is similar to an argument in Vaughan [14], but extra care is needed to save logarithms, and it is for this reason that details have been provided. 
3. We are ready to advance to Theorem 1. By orthogonality, one has

$$
r(m)=\int_{0}^{1} f_{2}(\alpha) f_{5}(\alpha)^{3} e(-\alpha m) \mathrm{d} \alpha
$$

for all $m \leq n$. For such integers $m$ and $\mathfrak{U}=\mathfrak{M}$ or $\mathfrak{m}$, let

$$
r(m, \mathfrak{U})=\int_{\mathfrak{U}} f_{2}(\alpha) f_{5}(\alpha)^{3} e(-\alpha m) \mathrm{d} \alpha
$$

so that

$$
r(m)=r(m, \mathfrak{M})+r(m, \mathfrak{m}) .
$$

The evaluation of $r(m, \mathfrak{M})$ is along well-trodden paths, so we shall be brief. Let

$$
S_{l}(q, a)=\sum_{x=1}^{q} e\left(\frac{a x^{l}}{q}\right), \quad v_{l}(\beta)=\frac{1}{l} \sum_{m \leq n} m^{\frac{1}{7}-1} e(\beta m) .
$$

Repeated use of [16, Lemma 2.7] shows that for $\alpha=\frac{a}{q}+\beta$ with $0 \leq a \leq q,(a ; q)=1$, $q \leq n^{\delta}$ and $|\beta| \leq n^{\delta-1}$ one has

$$
f_{2}(\alpha) f_{5}(\alpha)^{3}=q^{-4} S_{2}(q, a) S_{5}(q, a)^{3} v_{2}(\beta) v_{5}(\beta)^{3}+O(n) .
$$

One multiplies with $e(-\alpha m)$ and integrates to see that

$$
r(m, \mathfrak{M})=\sum_{q \leq n^{\delta}} A(q, m) J(m, n)+O\left(n^{3 \delta}\right)
$$

where

$$
A(q, m)=q^{-4} \sum_{\substack{a=1 \\(a ; q)=1}}^{q} S_{2}(q, a) S_{5}(q, a)^{3} e\left(-\frac{a m}{q}\right)
$$

and

$$
J(m, n)=\int_{-n^{\delta-1}}^{n^{\delta-1}} v_{2}(\beta) v_{5}(\beta)^{3} e(-\beta m) \mathrm{d} \beta .
$$

In the last integral, we extend the integration to $\left[-\frac{1}{2}, \frac{1}{2}\right]$. By [16, Lemma 2.8], the resulting error is $O\left(n^{\frac{1}{10}(1-\delta)}\right)$. Consequently, by orthogonality,

$$
J(m, n)=\frac{1}{250} \sum m_{1}^{-\frac{1}{2}}\left(m_{2} m_{3} m_{4}\right)^{-\frac{4}{5}}+O\left(n^{\frac{1}{10}(1-\delta)}\right) .
$$

where the sum on the right hand side is over all natural numbers $m_{1}, m_{2}, m_{3}, m_{4}$ satisfying $m_{j} \leq n$ and $m_{1}+m_{2}+m_{3}+m_{4}=m$. For $m \leq n$, the conditions of summation on $m, \ldots, m_{4}$ do not depend on $n$, and one may apply [16, Lemma 2.9] and the functional equation for the Gamma function repeatedly to deliver

$$
J(m, n)=\frac{\Gamma\left(\frac{3}{2}\right) \Gamma\left(\frac{6}{5}\right)^{3}}{\Gamma\left(\frac{11}{10}\right)} m^{\frac{1}{10}}+O\left(n^{\frac{1}{10}-\eta}\right) \quad(1 \leq m \leq n) .
$$


The treatment of the singular series is more subtle. We require the following lemma that we prove in the next section.

LEMMA 2. For natural numbers $m$ one has

$$
\sum_{q=1}^{\infty} q^{\delta}|A(q, m)| \ll m^{\varepsilon}
$$

In particular, the sum on the left converges.

Equipped with this lemma, we find that the singular series

$$
\mathfrak{s}(m)=\sum_{q=1}^{\infty} A(q, m)
$$

converges absolutely, and that one has $\mathfrak{s}(m) \ll m^{\varepsilon}$. Further, partial summation yields

$$
\sum_{q \leq n^{\delta}} A(q, m)=\mathfrak{s}(m)+O\left(n^{\varepsilon-\eta}\right)
$$

uniformly for $m \leq n$. For these $m$, we now infer from (12) and (10) that

$$
r(m, \mathfrak{M})=\frac{\Gamma\left(\frac{3}{2}\right) \Gamma\left(\frac{6}{5}\right)^{3}}{\Gamma\left(\frac{11}{10}\right)} \mathfrak{s}(m) m^{\frac{1}{10}}+O\left(n^{\frac{1}{10}-\eta+\varepsilon}\right),
$$

whence

$$
\sum_{m \leq n}\left|r(m, \mathfrak{M})-\frac{\Gamma\left(\frac{3}{2}\right) \Gamma\left(\frac{6}{5}\right)^{3}}{\Gamma\left(\frac{11}{10}\right)} \mathfrak{s}(m) m^{\frac{1}{10}}\right|^{2} \ll n^{\frac{6}{5}-\eta}
$$

Also, since the $r(m, \mathfrak{m})$ are the Fourier coefficients of the function that equals $f_{2}(\alpha) f_{5}(\alpha)^{3}$ on $\mathfrak{m}$ and that vanishes on $\mathfrak{M}$, an application of Bessel's inequality together with Lemma 1 shows

$$
\sum_{m \leq n}|r(m, \mathfrak{m})|^{2} \leq \int_{\mathfrak{m}}\left|f_{2}(\alpha) f_{5}(\alpha)^{3}\right|^{2} \mathrm{~d} \alpha \ll n^{\frac{6}{5}} L^{\varepsilon-1} .
$$

The desired estimate (2) is now available from (9), (14) and (15).

4. The treatment of the singular series and the proof of Lemma 2 require a careful analysis of the sum $A(q, m)$.

LEMMA 3. The sum $A(q, m)$ is multiplicative in $q$, and the bound

$$
A(q, m) \ll q^{-\frac{1}{10}}
$$

holds uniformly for natural numbers $m$. If $p$ is a prime with $p \geq 7$, and $t \in$ $\{1,2,3,6,7,8,9\}$, then

$$
A\left(p^{t}, m\right) \ll p^{-\frac{3}{2}}
$$


while if $t=4$ or $t=10$ one has $\left|A\left(p^{t}, m\right)\right| \leq p^{-2}$ except when $p^{t} \mid m$ where

$$
A\left(p^{t}, m\right)=\frac{p-1}{p^{2}} .
$$

Further, one has $A\left(p^{5}, m\right)=0$ unless $p^{4} \mid m, p^{5} \nmid m$ where

$$
A\left(p^{5}, m\right)=\left(\frac{m p^{-4}}{p}\right) \frac{1}{p} .
$$

For a proof, we first inspect the argument underpinning the demonstration of Lemma 2.11 in Vaughan [16], and see that it delivers the multiplicativity of $A(q, m)$. Then we recall that whenever $(a ; q)=1$ one has

$$
q^{-1} S_{l}(q, a) \ll q^{-\frac{1}{l}}
$$

([16, Theorem 4.2]). By (11), this implies (16).

The proof of (17) splits into many different cases. First suppose that $p \equiv 1 \bmod 5$. Then, there are exactly four non-principal Dirichlet characters modulo $p$, say $\chi_{1}, \chi_{2}, \chi_{3}, \chi_{4}$, with $\chi_{j}^{5}$ principal. For $p \nmid a$, Lemma 4.3 of Vaughan [16] asserts that

$$
S_{5}(p, a)=\sum_{j=1}^{4} \bar{\chi}_{j}(a) \tau\left(\chi_{j}\right)
$$

where $\tau(\chi)$ denotes the Gauß sum of the character $\chi$. Similarly, with $\tau_{p}$ the Gauß sum of the Legendre symbol modulo $p$, one has

$$
S_{2}(p, a)=\left(\frac{a}{p}\right) \tau_{p}
$$

By (11), it follows that

$$
A(p, m)=\frac{\tau_{p}}{p^{4}} \sum_{i, j, k=1}^{4} \tau\left(\chi_{i}\right) \tau\left(\chi_{j}\right) \tau\left(\chi_{k}\right) \sum_{a=1}^{p-1}\left(\frac{a}{p}\right) \bar{\chi}_{i} \bar{\chi}_{j} \bar{\chi}_{k}(a) e\left(-\frac{a m}{p}\right)
$$

Note that the product of the Legendre symbol with $\bar{\chi}_{i} \bar{\chi}_{j} \bar{\chi}_{k}$ is a non-principal character modulo $p$, whatever the choice of $i, j, k$. Hence, when $p \mid m$, one has $A(p, m)=0$. When $p \nmid m$, one may substitute $a m$ for $a$ to see that, up to multiplication with some complex number of modulus 1 , the inner sum over $a$ is the Gauß sum of the product of the Legendre symbol with $\bar{\chi}_{i} \bar{\chi}_{j} \bar{\chi}_{k}$. The modulus of all Gauß sums involved is $p^{\frac{1}{2}}$, so that $|A(p, m)| \leq 4^{3} p^{-\frac{3}{2}}$.

Next suppose that $p \not \equiv 1$ mod 5. Then Lemma 4.3 of Vaughan [16] gives $S_{5}(p, a)=$ 0 whenever $p \nmid a$, so that $A(p, m)=0$. This proves (17) when $t=1$.

Now consider $A\left(p^{t}, m\right)$ when $t=2$ or 3. Then, by [16, Lemma 4.4], one has $S_{5}\left(p^{t}, a\right)=p^{t-1}$ whenever $p \nmid a$. By (11) and (20) with $l=2$, we deduce (17), uniformly in $m$. A similar argument applies when $7 \leq t \leq 9$. In these cases, [16, Lemma 4.4] yields $S_{5}\left(p^{t}, a\right)=p^{t-2}$ for $p \nmid a$, and (17) follows from (11) and (20) with $l=2$. 
This leaves the case $t=6$. Here a combination of Lemmata 4.3 and 4.4 of Vaughan [16] gives $S_{5}\left(p^{6}, a\right) \ll p^{\frac{9}{2}}$. Once again, (17) then follows via (11) and (20) with $l=2$. This establishes (17) in all cases.

Next consider $A\left(p^{4}, m\right)$. By $\left[\mathbf{1 6}\right.$, Lemma 4.4], we have $S_{2}\left(p^{4}, a\right)=p^{2}$ and $S_{5}\left(p^{4}, a\right)=$ $p^{3}$ for all $p \nmid a$. Hence

$$
A\left(p^{4}, m\right)=p^{-5} \sum_{\substack{a=1 \\ p \nmid a}}^{p^{4}} e\left(-\frac{a m}{p^{4}}\right) .
$$

If $p^{4} \mid m$, this gives (18), and if $p^{4} \nmid m$, the inequality (5) produces $\left|A\left(p^{4}, m\right)\right| \leq p^{-2}$, as required.

The case $t=10$ is similar. Here [16, Lemma 4.4] yields $S_{2}\left(p^{10}, a\right)=p^{5}$ and $S_{5}\left(p^{10}, a\right)=p^{8}$ for all $p \nmid a$. Hence

$$
A\left(p^{10}, m\right)=p^{-11} \sum_{\substack{a=1 \\ p \nmid a}}^{p^{10}} e\left(-\frac{a m}{p^{10}}\right),
$$

and the conclusions concerning $A\left(p^{10}, m\right)$ follow as in the case $t=4$.

Finally, consider the case $t=5$. By Lemma 4.4 of Vaughan [16], whenever $p \nmid a$, one has

$$
S_{5}\left(p^{5}, a\right)=p^{4}, \quad S_{2}\left(p^{5}, a\right)=\left(\frac{a}{p}\right) p^{2} \tau_{p}
$$

Hence,

$$
A\left(p^{5}, m\right)=\frac{\tau_{p}}{p^{6}} \sum_{\substack{a=1 \\ p \nmid a}}^{p^{5}}\left(\frac{a}{p}\right) e\left(-\frac{a m}{p^{5}}\right) .
$$

If $p^{5} \mid m$ then the sum over $a$ vanishes so that $A\left(p^{5}, m\right)=0$. If $p^{5} \nmid m$ then we substitute $a=b+p c$ to see that

$$
\sum_{\substack{a=1 \\ p \nmid a}}^{p^{5}}\left(\frac{a}{p}\right) e\left(-\frac{a m}{p^{5}}\right)=\sum_{b=1}^{p-1}\left(\frac{b}{p}\right) e\left(-\frac{b m}{p^{5}}\right) \sum_{c=1}^{p^{4}} e\left(-\frac{c m}{p^{4}}\right) .
$$

Here the sum over $c$ again vanishes unless $p^{4} \mid m$ in which case the sum in the previous display equals

$$
p^{4} \sum_{b=1}^{p-1}\left(\frac{b}{p}\right) e\left(-\frac{b m^{\prime}}{p}\right)
$$

where the natural number $m^{\prime}=m p^{-4}$ is prime to $p$. The substitution $b m^{\prime}$ for $b$ shows that the sum over $b$ equals $\left(m^{\prime} \mid p\right) \bar{\tau}_{p}$, so that, on collecting together, we find that in this last situation one has $A\left(p^{5}, m\right)=\left(m^{\prime} \mid p\right) p^{-1}$. This completes the proof of Lemma 3 . 
The proof of Lemma 2 is also swiftly completed. By (16), the series

$$
E_{p}(m)=\sum_{t=0}^{\infty} p^{t \delta}\left|A\left(p^{t}, m\right)\right|
$$

converges absolutely, and on combining the conclusions in (16) for $q=p^{t}, t \geq 11$, with those in (17)-(19), one finds that $E_{p}(m)=1+O\left(p^{-\frac{21}{20}}\right)$ holds in all cases where $p^{4} \nmid m$. When $p^{4} \mid m$, a crude use of (16)-(19) gives $E_{p}(m) \leq 1+C p^{-\frac{2}{3}}$ for a suitable positive constant $C$. Using multiplicativity, we conclude that for any $Q \geq 1$ one has

$$
\sum_{q \leq Q} q^{\delta}|A(q, m)| \leq \prod_{p \leq Q} E_{p}(m) \ll \prod_{p^{4} \mid m}\left(1+\frac{C}{p^{\frac{2}{3}}}\right) .
$$

This implies Lemma 2.

It remains to establish the inequalities for $\mathfrak{s}(m)$ stated in Theorem 1 . By (13) and multiplicativity, one has

$$
\mathfrak{s}(m)=\prod_{p} A_{p}(m)
$$

where

$$
A_{p}(m)=\sum_{t=0}^{\infty} A\left(p^{t}, m\right)
$$

For an upper bound, we can argue much as in the preceding paragraph to obtain the estimate

$$
\mathfrak{s}(m) \ll \prod_{p^{4} \mid m}\left(1+\frac{3}{p}\right) \ll(\log \log m)^{3},
$$

as required. For a lower bound, first observe that $A_{p}(m)$ is real and non-negative. This is readily established by an adaptation of the arguments on p. 21 of Vaughan [16]. We then begin with (16) for $q=p^{t}, t \geq 11$, and use (17) to see that

$$
A_{p}(m)=1+A\left(p^{4}, m\right)+A\left(p^{5}, m\right)+A\left(p^{10}, m\right)+O\left(p^{-\frac{11}{10}}\right) .
$$

When $p^{4} \mid m$ it follows from Lemma 3 that $A\left(p^{4}, m\right)+A\left(p^{5}, m\right) \geq-p^{-2}$, and hence that

$$
A\left(p^{4}, m\right)+A\left(p^{5}, m\right)+A\left(p^{10}, m\right) \geq-2 p^{-2} .
$$

When $p^{4} \nmid m$, the inequality (21) is also valid, as one again finds from Lemma 3 . We may now conclude that there is a positive constant $C$ with the property that $A_{p}(m) \geq 1-C p^{-\frac{11}{10}}$ holds for all primes $p$. It follows that there is another positive number $c$ such that

$$
\prod_{p>c} A_{p}(m) \geq \frac{1}{2}
$$


To handle the primes $p$ with $p \leq c$, we note that for all primes $p \neq 5$, the congruence

$$
x^{2}+y_{1}^{5}+y_{2}^{5}+y_{3}^{5} \equiv m \bmod p
$$

has a solution with $p \nmid y_{1}$. This follows from a repeated application of the CauchyDavenport theorem ([16, Lemma 2.14]). When $p=5$, the congruence (23) has a solution with $p \nmid x$, as one easily checks. Again by an argument similar to the one leading to Lemma 2.13 of Vaughan [16], one then concludes that $A_{p}(m) \geq p^{-3}$. By (22), it follows that $\mathfrak{s}(m) \gg 1$. The proof of Theorem 1 is complete.

5. The proof of Theorem 2 is considerably simpler. By orthogonality,

$$
R(n)=\int_{0}^{1} f_{2}(\alpha)^{2} f_{5}(\alpha)^{6} e(-\alpha n) \mathrm{d} \alpha,
$$

and Lemma 1 immediately yields

$$
R(n)=\int_{\mathfrak{M}} f_{2}(\alpha)^{2} f_{5}(\alpha)^{6} e(-\alpha n) \mathrm{d} \alpha+O\left(n^{\frac{6}{5}} L^{\varepsilon-1}\right) .
$$

In the new context, the singular series is

$$
\mathfrak{S}(n)=\sum_{q=1}^{\infty} \sum_{\substack{a=1 \\(a ; q)=1}}^{q} q^{-8} S_{2}(q, a)^{2} S_{5}(q, a)^{6} e\left(-\frac{a n}{q}\right),
$$

and (20) shows that this sum converges absolutely and uniformly with respect to $n$. Moreover one finds that

$$
\sum_{q \leq n^{\delta}} \sum_{\substack{a=1 \\(a ; q)=1}}^{q} q^{-8} S_{2}(q, a)^{2} S_{5}(q, a)^{6} e\left(-\frac{a n}{q}\right)=\mathfrak{S}(n)+O\left(n^{-\frac{\delta}{5}}\right) .
$$

Hence, there is no difficulty to mimic the treatment of the major arcs in section 3 , the details being so much simpler, and with no need for a subtle analysis as in the proof of Lemma 3, that there is no need for a presentation here. Also, the lower bound for $\mathfrak{S}(n)$ is much easier to obtain than the one for $\mathfrak{s}(n)$, and we spare the details.

\section{REFERENCES} 233-243.

1. J. Brüdern, Sums of squares and higher powers. I. J. London Math. Soc. (2) 35(2) (1987),

2. J. Brüdern, A problem in additive number theory, Math. Proc. Cambridge Philos. Soc. 103(1) (1988), 27-33.

3. J.Brüdern and K. Kawada, The asymptotic formula in Waring's problem for one square and seventeen fifth powers, Monatsh. Math. 162(4) (2011), 385-407.

4. H. Davenport and H. Heilbronn, On Waring's problem: two cubes and one square, Proc. London Math. Soc. (2) 43(1) (1937), 73-104.

5. J.B. Friedlander and T.D. Wooley, On Waring's problem: Two squares and three biquadrates, Mathematika 60(1) (2014), 153-165.

6. G.H. Hardy and E.M. Wright, An introduction to the theory of numbers, 5th ed. (The Clarendon Press, Oxford University Press, New York, 1979).

7. Hooley $\mathrm{C}$, On the representations of a number as the sum of two cubes, Math. Z. 82 (1963), 259-266. 
8. C. Hooley, On a new approach to various problems of Waring's type, Recent progress in analytic number theory, vol. 1 (Durham, 1979) (Academic Press, London-New York, 1981), $127-191$.

9. C. Hooley, On Waring's problem for two squares and three cubes, Quart. J. Math. Oxford Ser. (2) 16 (1965), 289-296.

10. J. St.-C. L. Sinnadurai, Representation of integers as sums of six cubes and one square, Quart. J. Math. Oxford Ser. 16(2) (1965), 289-296.

11. G.K. Stanley, The representation of a number as the sum of one square and a number of $k$-th powers, Proc. London Math. Soc. (2) 31(1) (1930), 512-553.

12. R.C. Vaughan, On the representation of numbers as sums of squares, cubes and fourth powers, PhD Thesis (London, 1969).

13. R.C. Vaughan, A ternary additive problem, Proc. London Math. Soc. (3) 41(3) (1980), $516-532$

14. R.C. Vaughan, On Waring's problem: One square and five cubes, Quart. J. Math. Oxford Ser. (2) 37(145) (1986), 117-127.

15. R.C. Vaughan, On Waring's problem for smaller exponents. II, Mathematika 33(1) (1986), 6-22.

16. R.C. Vaughan, The Hardy-Littlewood method, 2nd ed. Cambridge Tracts in Mathematics, vol. 125 (Cambridge University Press, Cambridge, 1997).

17. R.C. Vaughan, On generating functions in additive number theory. I, Analytic number theory (Cambridge University Press, Cambridge, 2009), 436-448.

18. T.D. Wooley, On Waring's problem: Some consequences of Golubeva's method, J. Lond. Math. Soc. (2) 88(3) (2013), 699-715. 